\title{
A Fuzzy-Based Approach for Evaluating Existing Spatial Layout Configurations
}

\author{
Ayman Assem ${ }^{1}$, Sherif Abdelmohsen ${ }^{2}$, Mohamed Ezzeldin ${ }^{3}$ \\ ${ }_{1,3}$ Ain Shams University ${ }^{2}$ American University in Cairo; Ain Shams University \\ ${ }^{1,3}$ \{ayman.assem|mohamed.ezzeldin\}@eng.asu.edu.eg ${ }^{2}$ sherifmorad@aucegypt. \\ $e d u$
}

\begin{abstract}
This paper proposes a fuzzy-based approach for the automated evaluation of spatial layout configurations. Our objective is to evaluate soft and interdependent design qualities (such as connectedness, enclosure, spaciousness, continuity, adjacency, etc.), to satisfy multiple and mutually inclusive criteria, and to account for all potential and logical solutions without discarding preferable, likely or even less likely possible solutions. Using fuzzyTECH, a fuzzy logic software development tool, we devise all possible spatial relation inputs affecting physical and non-physical outputs for a given space using descriptive rule blocks. We implement this fuzzy logic system on an existing residential space to evaluate different layout alternatives. We define all linguistic input variables, output variables, and fuzzy sets, and present space-space relations using membership functions. We use the resulting database of fuzzy agents to evaluate the design of the existing residential spaces.
\end{abstract}

Keywords: Fuzzy logic, Space layout planning, Heuristic methods

\section{INTRODUCTION}

Several approaches have been proposed for evaluating and generating spatial layout configurations, including graph theory, quadratic assignment problems, slicing tree, space filling curves, genetic algorithms, and evolutionary approaches (Dunker et al., 2003; El-Baz, 2004; Aiello et al., 2012; Buscher et al., 2012). Heuristic methods to address space layout planning problems and allocation of objects within spatial configurations have been developed within several engineering disciplines and include greedy algorithms, branch and bound methods, dynamic programming, and single-solution metaheuristic methods such as tabu search (Ahuja et al.
2000; Xie and Sahinidis, 2008; Abdinnour-Helm \& Hadley 2000; Merrell et al., 2011; Yu et al., 2011; Abdelmohsen et al., 2017).

These approaches mostly involved computing distances between spatial configurations using graph algorithms and genetic algorithms, the hierarchical organization of layout elements, and multi-level space allocation using hybrid evolutionary techniques. Qualitative aspects of spatial configurations, however, related to ambiguities of space use and occupational behavior, ill-defined layout design and spatial uncertainty remain relatively unaddressed. Fuzzy logic systems have recently been proposed to address ambiguity in architectural de- 
sign approaches and requirements, and specifically in managing uncertainty and soft qualities in spatial layout design (Ciftcioglu \& Durmisevic, 2001; Çekmis, 2014; Çekmis, 2016). Most of these proposals attempted to address spatial layout design from an occupancy-centered perspective, where possibilities of occupancy are identified in ambiguously defined spaces or usage patterns, especially in open plan designs.

This paper proposes a fuzzy-based approach for the automated evaluation of spatial layout configurations by conducting an analysis of spatial parameters and identifying the nature of spatial relations using fuzzy logic. Our heuristic approach involves analyzing spatial characteristics and space-space relations to assess soft qualities such as connectedness, spaciousness, convenience of access, continuity, adjacency, etc. for a given layout scheme. We identify rulesets for logical configurations based on these relations. We put forward that the resulting range of possible configurations satisfy an intuitive process without the need for an exhaustive search through all possible solutions, but simultaneously include partially true assumptions and solutions that reflect how architects would perceive given spatial configurations based on intuitive logic.

\section{SPATIAL RELATIONS AND FUZZY LOGIC}

Traditional scenarios of space layout planning involve defining preset holistic notions of relations between spaces that dictate their adjacency and configuration logic. For example, a "strong" or "weak" relationship between two spaces is roughly defined in conventional spatial relation matrix diagrams based on universal understandings of connectivity, adjacency, proximity and visibility. These relationships are typically defined generically and may discard (a) a wide spectrum of parameters and variables that affect spatial relations, (b) detailed relations between input spatial parameters and spatial relation outputs, and (c) the inclusion of partially true solutions for spatial configurations.

In this context, our approach deals with soft computing methodologies (Ciftcioglu \& Durmisevic, 2001; Bittermann, 2009). Rather than well defined design issues, our approach is more concerned with (a) the understanding and evaluation of soft and highly interdependent aspects of design, (b) the satisfaction of multiple and mutually inclusive criteria simultaneously including functional, aesthetic, environmental, and spatial issues, and (c) the consideration of both technical and non-technical aspects of design, such as visibility, intimacy, adjacency, connectedness, enclosure, spaciousness and accessibility, (d) accounting for all potential and logical design solutions and alternatives that encompass a wide variety of parameters, without discarding preferable, likely or even less likely possible solutions.

Fuzzy logic approaches are based, as opposed to classical two-valued logic that assumes only true or false propositions, on propositions that may be both partially true and partially false (Figure 1).
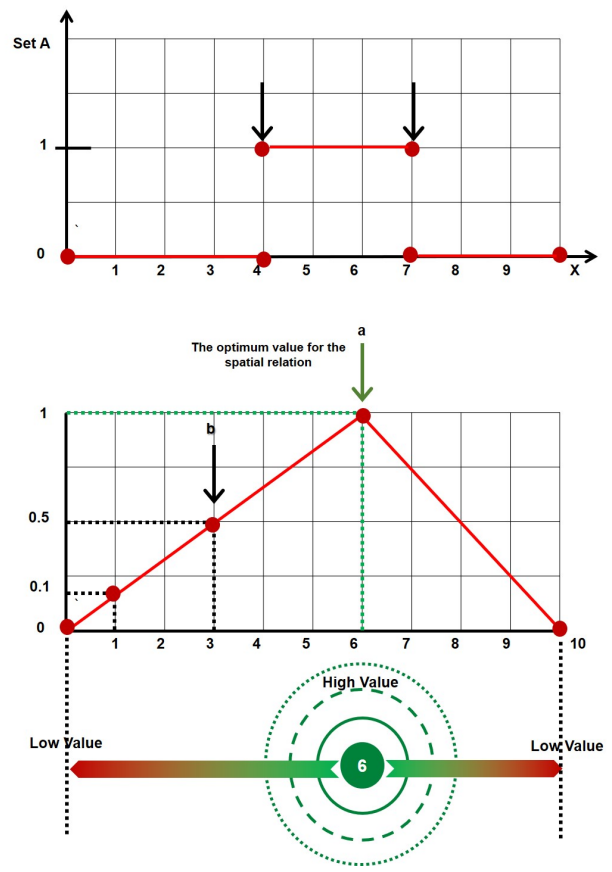

Figure 1

Difference between classical and fuzzy spatial relation input variables (Top: Classical set, Bottom: Spatial relation input variables using fuzzy curves) 
Figure 2

Example of using

fuzzy rules in

controlling the relation between spatial inputs and output

Figure 3

The influence of a spatial relation input variable on an output variable
The classical set demonstrates one and only truth value for a finite number of logical variables. In fuzzy logic however, input variables are passed into the fuzzy logic system as a fuzzy variable, presented as a vector of membership degrees, as they stem originally from a qualitative or linguistic source. The $X$-axis represents alternatives of the factor under study; for example, proximity, while the $\mathrm{Y}$-axis represents the fuzzy degree which lies between 0 and 1. For example, point $a$ in the figure represents the highest desired value of the fuzzy curve, while $(0,0)$ and $(10,0)$ represent the lowest. All other points represent values that are desired and not desired at the same time with varying degrees (e.g. point $b$ in the figure lies on the curve where the value is 50\% desired and $50 \%$ undesired simultaneously).

\section{APPROACH}

We propose a fuzzy logic approach whereby spatial inputs (e.g. space area, space proportion, viewing angles, sum of openings per space, etc.) that affect specific outputs (e.g. adjacency, spaciousness, connectedness, etc.) are identified for a given space. Based on the fuzzy curves for each of the input variables, we devise a rule block which comprises the control strategy of the fuzzy logic system and linguistically defines the relation between inputs and outputs as if-then equations. These equations describe the situation for which the rules are designed and the response of the fuzzy system for that specific situation, as shown in Figure 2. Consequently, a finite yet extensive set of specific relations can be identified for each of the descriptive outputs that contribute to the evaluation of physical and non-physical attributes of a given space. Using fuzzyTECH, a software development tool for fuzzy logic and neural-fuzzy solutions, we devise all possible spatial relation inputs affecting physical and non-physical outputs for a given space using descriptive rule blocks, describing the fuzzification and defuzzification processes. As a case study, we implement this fuzzy logic system on an existing residential space to evaluate spatial layout configuration alternatives. We define some space-space rela- tions and present these relations using fuzzy curves. We define all linguistic input variables, output variables, and fuzzy sets (e.g. high, medium, low).

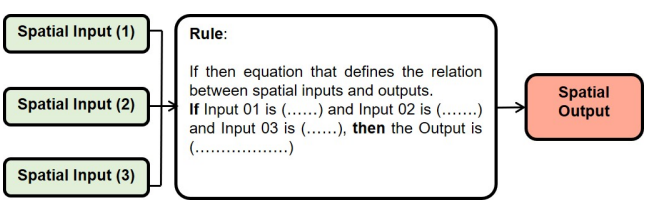

By specifying the influence of input variables on the output variable (Figure 3), the rule block wizard in the software uses this data to determine the necessary rulesets in (If-Then) equation format. We use the resulting database of fuzzy agents to evaluate the design of the existing residential building.

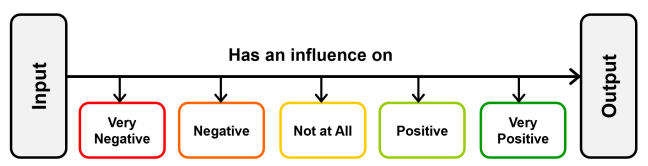

In the sections below, we introduce operational definitions and notations for space entities, and a detailed account of the proposed spatial relation inputs and outputs, and the fuzzy logic inference flow from input variables to output variables using rule blocks containing the linguistic control rules.

\section{Definition of Space Entities}

In order to introduce the spatial relation inputs and outputs, we present first some basic definitions and notations for spatial entities and their variables which are seen to inform space-space relations in the fuzzification and defuzzification process. For a given space (Sn), we identified the following sets of entities and their notations, as shown in Figure 4:

Set 1: Space Dimensions and Boundaries: This includes basic "Space Dimensions" ( $\left.X \_S n, Y \_S n\right)$, "Space Area" (A_Sn), "Center of Space" (C_Sn), "Corner Point of Space" (CRn_Sn), "Space Wall" (Wn_Sn), "Free Wall" (or external wall) (FWn_Sn), and "Viewing Angle from Center of Space" towards the exterior (CAn_Sn). 


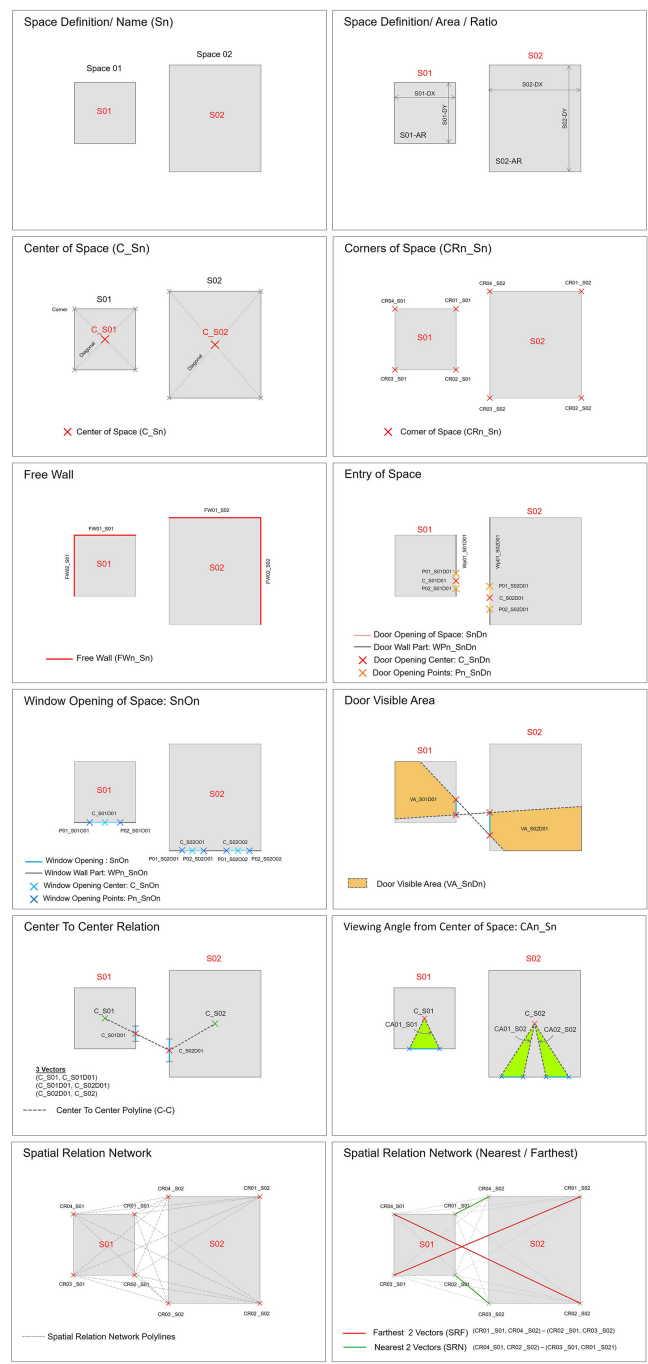

Set 2: Door Openings and Relations: This includes "Door Opening of Space" (SnDn), "Door Opening Points" (Pn_SnDn) denoting the start and end points for a given door opening, "Door Opening Center" ( $C_{-}$$S n D n)$, "Door Wall Part" (WPn_SnDn) which denotes the remaining wall segment in a door wall, and "Door
Visible Area" (VA_SnDn) which denotes the area in space visible through a given door opening.

Set 3: Window Openings and Relations: This includes "Window Opening of Space" (SnOn), "Window Opening Points" (Pn_SnOn) denoting the start and end points for a given window opening, "Window Opening Center" (C_SnOn), "Window Wall Part" (WPn_SnOn) which denotes the remaining wall segment in a window wall, and "Window Visible Area" (VA_SnOn) which denotes the area in space visible through a given window opening.

\section{Spatial Inputs}

Based on the space entity definitions above, we developed 16 linguistic variables for spatial inputs and their fuzzy sets and membership functions to initiate the fuzzification process. Different combinations of these inputs formulate rule blocks for defining a variety of spatial outputs for any given spatial layout configuration. Table 1 illustrates the codes developed for these inputs, their basic definition, calculation method, unit, and fuzzy sets or descriptors.

The spatial inputs IN01_Area_Sn, INO2_Proportion, and IN03_Number_FWn compute the area, proportion of space dimensions, and number of free walls per space respectively. Inputs IN04_Length_SnDn and IN05_Ratio_SnDn compute the length of a given door opening and its percentage in relation to wall lengths per space. Inputs INO6_Sum_SnOn and IN07_Ratio_SnOn compute the total window opening lengths and their percentage in relation to total wall lengths per space. Inputs IN08_Ratio_VA_SnDn and IN09_Ratio_VA_SnOn compute the ratio of door and window visible area per space respectively, whereas IN10_Sum_CAn_Sn computes the total angle of viewing from the center of a given space.

Inputs IN11_Length_CenterToDoorVector and IN12_Angle_CenterToDoorVector compute the distances and angles of the internal space vectors from center of space to door, while inputs IN13_Length_SpaceToSpaceVector and IN14_Angle_SpaceToSpaceVector compute the distances and angles of the external vectors from space door to space
Figure 4

Definition of space entities 
Table 1

Variables of spatial inputs

Figure 5

Membership

function graph for

spatial input

"IN02_Proportion"

Table 2

Definition points of membership

function for spatial input

"IN02_Proportion"

\begin{tabular}{|c|c|c|c|c|c|c|c|}
\hline CODE (Long) & ALTERNATE CODE (Short) & DEFINITION & CALCULATION METHOD & UNIT & \multicolumn{3}{|c|}{ FUZZY SETS } \\
\hline IN01_Area_Space & IN01_Area_Sn & Space Area & A_Sn & $\mathrm{m}^{2}$ & Small & Medium & Large \\
\hline IN02_Proportion_Space & IN02_Proportion & Proportion of space dimensions & (Y_Sn/X_Sn) & & Squarish & Rectangular & Linear \\
\hline IN03_Number_FreeWalls & INO3_Number_FWn & Number of free walls per space & $\Sigma\left(F W n \_S n\right)$ & & Low & Regular & High \\
\hline IN04_Length_DoorOpening & IN04_Length_SnDn & Length of door opening per space & L_SnDn & $\mathrm{m}$ & Narrow & Regular & Wide \\
\hline IN05_Ratio_DoorOpenings & INO5_Ratio_SnDn & Ratio of door openings to walls per space & $\left(\mathrm{L} \_\mathrm{SnDn}\right) / \Sigma\left(\mathrm{WPn} \_\mathrm{SnDn}\right)$ & Ratio & Low & Regular & High \\
\hline IN06_Sum_WindowOpenings & IN06_Sum_SnOn & Sum of window opening lengths per space & $\Sigma\left(L_{-}\right.$SnOn $)$ & $\mathrm{m}$ & Low & Medium & High \\
\hline IN07_Ratio_WindowOpenings & IN07_Ratio_SnOn & Ratio of window opening lengths to wall lengths & $\Sigma\left(L_{-}\right.$SnOn $) / \Sigma($ WPn_SnOn $)$ & Ratio & Low & Regular & High \\
\hline IN08_Ratio_DoorVisibleArea & IN08_Ratio_VA_SnDn & Ratio of door visible area per space & VA_SnDn/A_Sn & Ratio & Low & Medium & High \\
\hline IN09_Ratio_WindowVisibleArea & INO9_Ratio_VA_SnOn & Ratio of window visible area per space & $\Sigma\left(\mathrm{VA} \_\mathrm{SnOn}\right) / \mathrm{A} \_\mathrm{Sn}$ & Ratio & Low & Medium & High \\
\hline IN10_Sum_ViewingAngles & IN10_Sum_CAn_Sn & Sum of viewing angles from center of space & $\Sigma\left(\mathrm{CAn} \_\mathrm{Sn}\right)$ & $\varnothing$ & Low & Medium & High \\
\hline IN11_Length_CenterToDoorVecto & IN11_Length_[C_Sn,C_SnDn] & Length of center-to-door vector per space & $\|\left[C_{-} S n, C_{-}\right.$SnDn $] \|$ & $\mathrm{m}$ & Near & Regular & Far \\
\hline IN12_Angle_CenterToDoorVector & IN12_Angle_[C_Sn,C_SnDn] & Angle of center-to-door vector per space & $\tan -1(y / x)$ of vector & $\varnothing$ & Direct & & Indirect \\
\hline IN13_Length_SpaceToSpaceVecto & IN13_Length_[C_SnDn,C_SnDn] & Length of vector between space doors & $\|\left[C_{-}\right.$SnDn, C_SnDn] $\|$ & $\mathrm{m}$ & Near & Regular & Far \\
\hline IN14_Angle_SpaceToSpaceVector & IN14_Angle_[C_SnDn,C_SnDn] & Angle of vector between space doors & $\tan -1(y / x)$ of vector & $\varnothing$ & Direct & & Indirect \\
\hline IN15_Length_NearestVectors & IN15_Length_SRN & Average length of two nearest vectors between spaces & $\mu \mathrm{SRN}$ & $\mathrm{m}$ & Near & Regular & Far \\
\hline IN16_Length_FarthestVectors & IN16_Length_SRF & Average length of two farthest vectors between spaces & $\mu \mathrm{SRF}$ & $\mathrm{m}$ & Near & Regular & Far \\
\hline
\end{tabular}

door. Inputs IN15 Length SRN and IN16 Length SRF compute averages of the distances between the two nearest and farthest vectors between spaces, calculated from space corner points.

For each of the linguistic variables, we identified a set of descriptors or fuzzy sets (for example: low, medium, and high descriptors for space area; narrow, regular, and wide descriptors for door lengths; squarish, rectangular, and linear configuration descriptors for space proportion, etc.). Each of these descriptors describes the spatial input more or less well depending on the computed measurement or dimension. Each descriptor is defined by a membership function which highlights the associated degree of membership of the linguistic term for any value of the spatial input variable. The membership functions of all descriptors for one linguistic variable are typically shown in one collective graph. The graph in Figure 5 plots the membership functions of the descriptors for the variable IN02_Proportion, and Table 2 displays the definition points for each of the descriptors.

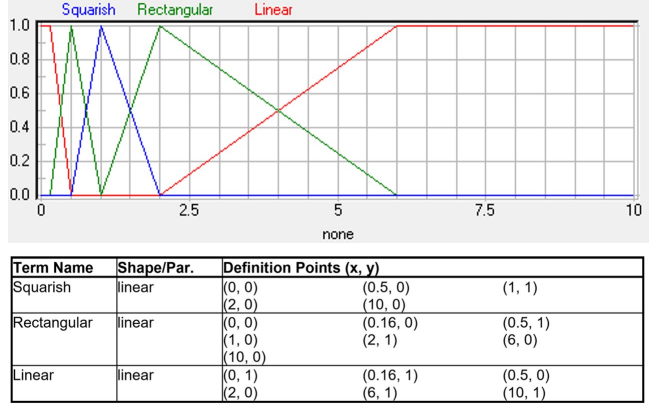

\section{Spatial Outputs}

We identified 10 linguistic variables for spatial outputs and their fuzzy sets and membership functions for the defuzzification process. Table 3 illustrates the codes developed for these outputs, their basic definition, unit, and fuzzy sets or descriptors. The 10 linguistic variables target primarily two sets of outputs: 1) outputs that describe individual space characteristics, including spaciousness, openness, intimacy, continuity, directionality, and enclosure, and 2) outputs that describe space-space relations, including adjacency, visibility, convenience of access, and connectedness. 


\begin{tabular}{|c|c|c|c|c|c|c|}
\hline CODE (Long) & ALTERNATE CODE (Short) & DEFINITION & UNIT & \multicolumn{3}{|c|}{ FUZZY SETS } \\
\hline OUT01_Spaciousness & OUT01_Spacious & Degree of abundance and sufficiency of space & $\%$ & Low & Medium & High \\
\hline OUT02_Openness & OUT02_Openness & Degree of exposure of space to external viewing and outdoor environment & $\%$ & Low & Medium & High \\
\hline OUT03_Intimacy & OUT03_Intimacy & Degree of privacy and intimate scale of space & $\%$ & Low & Medium & High \\
\hline OUT04_Continuity & OUT04_Continuity & Degree of free, consistent and unobstructed visual and physical flow of space & $\%$ & Low & Medium & High \\
\hline OUT05_Directionality & OUT05_Direction & Degree of proportionality and prevailing circulation in space & $\%$ & Low & Medium & High \\
\hline OUT06_Enclosure & OUT06_Enclosure & Degree of physical or virtual boundedness of space & $\%$ & Minimal & Partial & Maximum \\
\hline OUT07_Adjacency & OUT07_Adjacency & Degree of proximity and immediacy of space connection to other spaces & $\%$ & Weak & Regular & Strong \\
\hline OUT08_Visibility & OUT08_Visibility & Degree of space-to-space viewing range and visual access & $\%$ & Weak & Regular & Strong \\
\hline OUT09_AccessConvenience & OUT09_AccessConv & Degree of space-to-space physical accessibility & $\%$ & Low & Medium & High \\
\hline OUT10_Connectedness & OUT10_Connection & Degree of physical linkage of space to other spaces & $\%$ & Poor & Average & Good \\
\hline
\end{tabular}

Table 3

Variables for spatial outputs
Regarding the first set of outputs which describe individual space characteristics, OUTO1_Spacious denotes the degree of abundance and sufficiency of room per space; how spacious it is. OUT02_Openness denotes the degree of exposure of space to external viewing and the outdoor environment. OUTO3_Intimacy denotes the degree of privacy and intimate scale of space. OUTO4_Continuity denotes the degree of free and unobstructed flow within a given space, both physically and visually. OUT05_Directionality denotes the basic orientation and configuration of space in terms of prevailing circulation and proportionality. OUT06_Enclosure denotes the degree of physical or virtual boundedness and sense of enclosure of space. As for the second set of outputs which describe space-space relations, OUT07_Adjacency indicates the degree of proximity and immediacy of space connection to other neighboring spaces. OUT08_Visibility indicates the degree of visual access and viewing from one space to another. OUTO9_AccessConv indicates the degree and level of convenience and comfort of physical accessibility from one space to another. OUT10_Connectedness indicates the degree of physical connectivity and linkage of one space to multiple spaces.

For each of the linguistic variables of these spatial outputs, we identified a set of descriptors or fuzzy sets (e.g. low, medium, high for spaciousness; minimal, partial, maximum for enclosure; poor, average, strong for connectedness; weak, regular, strong for visibility and adjacency, etc.). Each descriptor is defined by a membership function which highlights the associated degree of membership of the linguistic term for any value of the spatial output variable. The graph in Figure 6 plots the membership functions of the descriptors for the variable INO2_Proportion, and Table 4 displays the definition points for each of the descriptors.

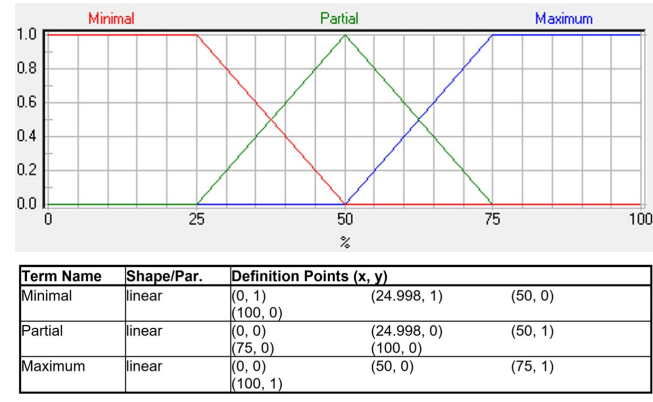

\section{Rule Blocks}

We identified 10 rule blocks for the proposed fuzzy logic system, whereby different combinations of spatial inputs contribute to the degree of satisfaction of a given spatial output. For example, for Rule Block 09 (RB09_AccessConv), as shown in Figure 7, if the descriptor for the input "IN13_Length_Space-
Figure 6

Membership function graph for spatial output "OUT06_Enclosure"

Table 4

Definition points of membership function for spatial output "OUT06_Enclosure" 
Figure 7

RB09_AccessConv:

Rule block for

controlling the

relation between

inputs $13 \& 14$ and

Output 09

Figure 8

Overall structure of proposed fuzzy

logic system
ToSpaceVector", which describes the length of the vector between the door centers for any two given spaces, is "Near", and the descriptor for the input "IN14_Angle_SpaceToSpaceVector", which describes the angle of that vector, is "Direct", then the descriptor for the output "OUTO9_AccessConv", which describes the degree of physical accessibility between those two spaces, is "High". By contrast, if the descriptor for the input "IN13_Length_SpaceToSpaceVector" is "Far", and that of the input "IN14_Angle_SpaceToSpaceVector" is "Indirect", then the descriptor for the output "OUT09_AccessConv" is "Low".

These are however not the only two possible combinations indicating a comprehensive evaluation of the degree of physical accessibility between those two spaces. Other possibilities include combinations of "Near", "Regular" and "Far" fuzzy sets or descriptors for the first spatial input and "Direct" and "Indirect" fuzzy sets for the second spatial input, which yield varying results for the "AccessConvenience" output, including "High", "Medium" or "Low" degrees.

For this specific rule block, 2 spatial inputs (IN13_Length_SpaceToSpaceVector and IN14_Angle_SpaceToSpaceVector) were identified for 1 spatial output (OUT09_AccessConv), with 6 rules describing the different conditions for evaluating the degree of access convenience. This typically varies for each rule block, depending on the number of involved spatial inputs, number and nature of descriptors, and the underlying rule controlling the relation between the spatial input(s) and spatial output.

The overall structure of the fuzzy logic system in its entirety is composed of 16 input variables, 10 output variables, 10 rule blocks that control the relations between spatial input and output variables, 2343 rules, and 79 membership functions. Figure 8 illustrates the overall structure of the proposed fuzzy logic system, and how the combinations of different spatial inputs formulate the final 10 spatial outputs using the developed rule blocks.

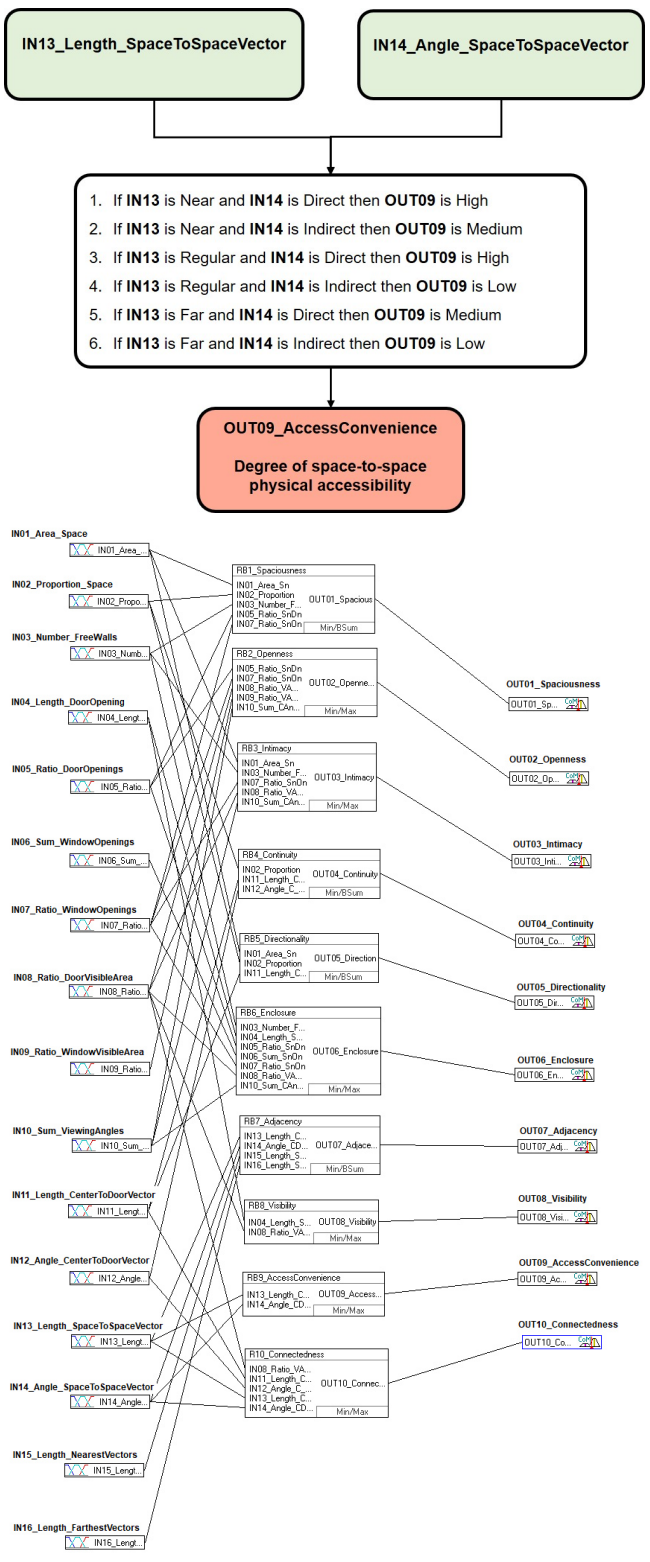




\section{CASE STUDY}

We introduce two cases that involve two residential spaces in close proximity and are connected through a circulation space, as shown in Figure 9. The two cases vary slightly in terms of their geometric configuration, area, space proportion, and door-to-door location. For each of the cases, we identified the basic space entity definitions (following the definitions developed earlier in Figure 4), including space area, ratio, center of space, corners of space, number of free walls, door opening length, door wall part length, door opening center, door opening points, window opening length, window wall part length, window opening center, window opening points, door visible area, space 01 center to space 02 center relations (including 3 vector lengths and angles), viewing angles from the centers of both spaces, and the spatial network relations between the two spaces computed from their corners, including the nearest vectors (SRN) and farthest vectors (SRF).
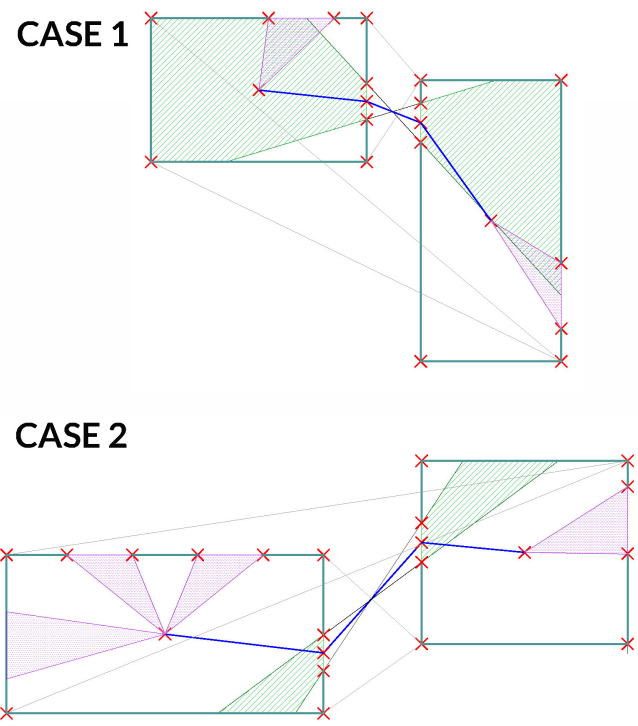

To initiate the fuzzification process, we computed all 16 spatial input parameters for both cases using
Grasshopper, including space area and proportion, number of free walls, length and ratio of door openings, sum and ratio of window openings, ratio of door and window visible area, sum of viewing angles from centers of both spaces, internal and external distances and angles of space-space vectors, and the average lengths of the nearest and farthest vectors between both spaces, computed from their corners. Table 5 shows the computed values for spatial inputs for both cases.

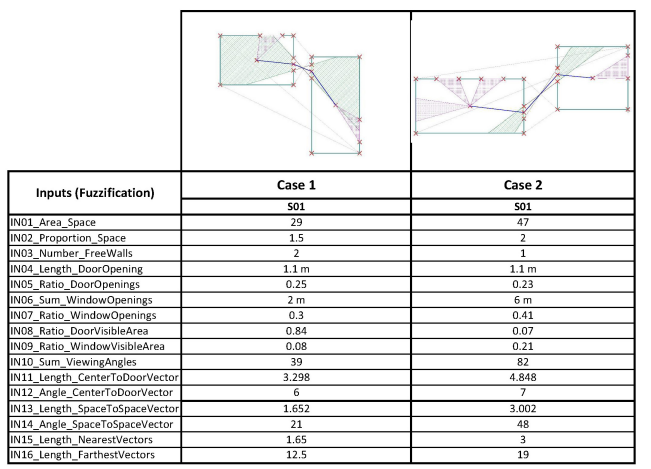

Using the fuzzyTECH fuzzy logic software, we used the developed rule block logic to translate the different spatial inputs and their fuzzy sets into the 10 spatial outputs, thereby resulting in an automated evaluation of the degrees of spaciousness, openness, intimacy, continuity, directionality, enclosure, adjacency, visibility, convenience of access, and connectedness for both cases, as shown in Table 6.

The results of the automated evaluation of the two spatial configuration cases demonstrate interesting findings. As shown in the table, subtle variations in the shape geometry of the two spaces can result in large discrepancies in the output variables. For example, the degree of visibility for case 1 was recorded at $93 \%$, as opposed to $7 \%$ in case 2 . Tracing this output back to its spatial input constituents, two main spatial inputs are shown to affect the degree of visibility: (1) IN04_Length_DoorOpenings and (2) IN08_Ratio_DoorVisibleArea. While the dooropening length remains the same in both cases (at $1.10 \mathrm{~m}$ ),
Table 5

Computed values for spatial inputs for the two residential cases (fuzzification)

Figure 9

Case study involving two spatial configuration cases 
Table 6

Results of automated evaluation of spatial outputs (defuzzification) the computed value for the door visible area was 0.84 for case 1 and 0.07 for case 2 .

\begin{tabular}{|c|c|c|c|c|}
\hline \multirow{2}{*}{ Outputs (Defuzzification) } & \multicolumn{2}{|r|}{ Case 1} & \multirow{2}{*}{\multicolumn{2}{|c|}{$\begin{array}{l}\text { Case 2 } \\
501: 502\end{array}$}} \\
\hline & & $501: 502$ & & \\
\hline out01_Spaciousness & $36 \%$ & & $53 \%$ & \\
\hline OUT02_Openness & $50 \%$ & & $16 \%$ & \\
\hline oUT03_Intimacy & $52 \%$ & & $50 \%$ & \\
\hline OUT04_Continuity & $63 \%$ & & $87 \%$ & \\
\hline ouT05_Directionality & $31 \%$ & & $56 \%$ & \\
\hline OUT06_Enclosure & $50 \%$ & & $50 \%$ & \\
\hline OUT07_Adjacency & $82 \%$ & & $36 \%$ & \\
\hline OUT08_Visibility & $93 \%$ & & $7 \%$ & \\
\hline OUTO9_AccessConvenience & $88 \%$ & & $37 \%$ & \\
\hline OUT10_Connectedness & $88 \%$ & & $40 \%$ & \\
\hline
\end{tabular}

Other revealing findings are related to the interesting dichotomies that can be deduced from such a result for the two cases, for example the degree of openness $(50 \%-16 \%)$ versus the degree of enclosure $(50 \%$ - $50 \%)$, the degree of spaciousness (36\% - 53\%) versus the degree of intimacy ( $52 \%-50 \%)$, the degree of continuity $(63 \%-87 \%)$ versus the degree of directionality $(31 \%-56 \%)$, the degree of spaciousness (36\% $53 \%)$ versus the degree of openness (50\% - 16\%). On the other hand, some values are in close correlation, such as the degree of convenience of access ( $88 \%$ $37 \%)$, the degree of adjacency $(82 \%-36 \%)$, and the degree of connectedness ( $88 \%-40 \%)$. These results, involving both the seemingly contradicting and the correlating, only corroborate the assumption of this research, where spatial relations cannot be evaluated in their totality as merely "strong" or "weak" relations, but are rather far more nuanced and involve a wide range of attributes that are more telling about the nature of spatial relations and configuration logic.

\section{DISCUSSION AND FUTURE WORK}

This paper introduced a fuzzy logic system for the automated evaluation of soft qualities of spatial configurations, including individual space characteristics and space-space relations. The findings of the paper confirm the initial assumption related to spatial ambiguity and uncertainty, where conventional spatial relation matrix diagrams tend to describe those relations in a holistic fashion as "strong" or "weak" relations, while discarding many of the nuances associated with soft qualities of space, such as spaciousness, intimacy, openness, enclosure, connectedness, etc. The paper attempted to evaluate these qualities by providing a detailed account of space entity definitions, calculation methods for spatial inputs, and devising rule blocks to address spatial outputs that take into account complex combinations of these spatial inputs and their fuzzy sets or descriptors.

The findings of this paper only open the door for extensive research in several areas pertaining to the automated evaluation of spatial configurations. One of the limitations of this research pertains to building typology. The discussed cases were relatively simple and arbitrary residential spaces. Other building typologies and spaces have their complexities and will affect the nature and understanding of the described outputs and rule blocks, and hence the nature of the evaluation process itself. Scale is also a significant factor and potential. The relations described in this paper, although demonstrated in the context of two simple residential rooms, can be applied in principle to different scales, levels of detail, and relations, including but not limited to furniture layout schemes in different spatial typologies, departmental spaces and zones, vertically stacked spaces in building configurations, urban neighborhoods and clusters, etc. This opens the door for studying a larger and more complex set of relations than only space-space re- 
lations, but also involves space-object and objectobject relations, and for evaluating alternative design schemes at different levels of detail, ranging in essence from rooms to cities.

Another significant and influential limitation pertains to relying solely on two-dimensional spatial configurations. Incorporating 3-dimensional and volumetric data for space layout configurations will definitely extend to include a larger dataset and become more inclusive and accurate when it comes to describe soft qualities such as spaciousness, enclosure, visibility, and the like. Further work is needs in this area to enhance the fuzzification process for inclusion of such dimensions. The studied geometries in this paper were also of average complexity, involving simple and primitive geometrical shapes, and basic orthogonal configurations. Geometrical complexity is yet another factor that will need further research, and will help refine and revisit the space entity definitions developed in this paper.

This paper has also focused in principle on physical qualities of space and relatively quantifiable attributes that can be tangibly reduced to geometrical entities. More research should focus on non-physical attributes, or non-physical components in the evaluation of soft qualities in space layout configurations, where notions such as perception and experience become incorporated into the understanding of seemingly mere physical qualities such as visibility, openness, continuity, intimacy, etc. Other dimensions such as light, material, texture, color, and the incorporation of environmental aspects should contribute to such an area of research.

As a long-term goal, findings from this research and future studies should eventually address generative aspects of design, where results of evaluating spatial layouts should inform architects with a more inclusive design process that includes partially true assumptions and solutions based on intuitive logic.

\section{REFERENCES}

Abdelmohsen, S, Assem, A, Tarabishy, S and Ibrahim, A 2017, 'A Heuristic Approach for the Automated Gen- eration of Furniture Layout Schemes in Residential Spaces', in Gero, J (eds) 2017, Design Computing and Cognition '16, Springer, pp. 459-475

Abdinnour-Helm, S and Hadley, SW 2000, 'Tabu Search based Heuristics for Multi-floor Facility Layout', The International Journal of Production Research, 38, pp. 365-383

Ahuja, RK, Orlin, JB and Tiwari, A 2000, 'A Greedy Genetic Algorithm for the Quadratic Assignment Problem', Computers \& Operations Research, 27, pp. 917-934

Aiello, G, La Scalia, G and Enea, M 2012, 'A Multi Objective Genetic Algorithm for the Facility Layout Problem based upon Slicing Structure Encoding', Expert Systems with Applications, 39, p. 10352-10358

Bittermann, MS 2009, Intelligent Design Objects (IDO): A Cognitive Approach for Performance-based Design, Ph.D. Thesis, TU Delft

Buscher, U, Mayer, B and Ehrig, T 2012, 'A Genetic Algorithm for the Unequal Area Facility Layout Problem', in Helber, S, Breitner, M, Rösch, D, Schön, C, Graf von der Schulenburg, J-M, Sibbertsen, $P$, Steinbach, $M$, Weber, S and Wolter, A (eds) 2012, Proceedings of Operations Research, Springer, Berlin, p. 109-114

Ciftcioglu, Ö and Durmisevic, S 2001 'Fuzzy Logic in Architectural Design', Proceedings of European Society for Fuzzy Logic and Technology (EUSFLAT) Conference

Dunker, T, Radons, G and Westkämper, E 2003, 'A Coevolutionary Algorithm for a Facility Layout Problem', The International Journal of Production Research, 41, p. $3479-3500$

El-Baz, MA 2004, 'A Genetic Algorithm for Facility Layout Problems of Different Manufacturing Environments', Computers \& Industrial Engineering, 47, p. 233-246

Merrell, P, Schkufza, E, Li, Z, Agrawala, M and Koltun, V 2011, 'Interactive Furniture Layout using Interior Design Guidelines', ACM Transactions on Graphics, 30(4), p. $87: 1-87: 9$

Xie, W and Sahinidis, NV 2008, 'A Branch-and-bound Algorithm for the Continuous Facility Layout Problem', Computers \& Chemical Engineering, 32, pp. 10161028

Yu, L-F, Yeung, SK, Tang, C-K, Terzopoulos, D, Chan, T and Osher, S 2011, 'Make it Home: Automatic Optimization of Furniture Arrangement', ACM Transactions on Graphics, 30(4), p. 86:1-86:11

Çekmis, A 2014 'Fuzzy Computing for Layout Design in III-defined, Uncertain Spaces', Proceedings of CAADe 2014, Newcastle upon Tyne, pp. 277-286

Çekmis, A 2016, 'Fuzzy Logic in Architectural Site Planning Design', Procedia Computer Science, 102, pp. 176-182 